\title{
SALARIOS, AUMENTOS E INCENTIVOS EN INSTITUCIONES DE SERVICIOS DE SALUD: CASO VILLAVICENCIO*
}

\author{
SALARIES, RAISES AND INCENTIVES IN HEALTH CARE \\ INSTITUTIONS: THE CASE OF VILLAVICENCIO (COLOMBIA) \\ SALÁRIOS, AUMENTOS E INCENTIVOS NAS INSTITUIÇÕES DE \\ SAÚDE: O CASO DO VILLAVICENCIO (COLOMBIA) \\ SALAIRES, AUGMENTATIONS ET INCITATIONS DANS LES
ETABLISSEMENTS DE SANTE: LE CAS DE VILLAVICENCIO \\ (COLOMBIE) \\ DAGOBERTO TORRES-FLÓREZ† \\ ANDREY CAMILO LAVERDE FORERO‡
}

Fecha de recepción: 8 de julio de 2020

Fecha de aprobación: 15 de octubre de 2020

RESUMEN

Realizar un análisis de las compensaciones salariales resulta ser un ejercicio muy beneficioso para aportar a la mejora de las empresas, ya que permite conocer la situación actual y determinar qué se está haciendo mal y, por tanto, conocer estas características que presentan las instituciones prestadoras de servicios de salud, IPS, de Villavicencio, permitió conocer los elementos salariales y de contratación que aplican las IPS a sus colaboradores, además de identificar los beneficios e incentivos con los que intentan motivar y retener a sus colaboradores, y determinar si se tiene en cuenta lo

* Resultados del proyecto de investigación "Análisis de los procesos de gestión humana en IPS de Villavicencio", financiado por la Universidad de los Llanos.

† Administrador público, ESAP Villavicencio, Colombia; administrador de empresas (E), Universidad Cooperativa de Colombia, Villavicencio; especialista en Gerencia de Recursos Humanos, Universidad Jorge Tadeo Lozano, Bogotá, Colombia; magíster en Administración de Empresas, Universidad del Mar, Viña del Mar, Chile; doctorando en Ciencias Económico Administrativas, Universidad para la Cooperación Internacional, UCIMEXICO, Quintana Roo, México; líder del Grupo de Investigación GYDO. dtorres@unillanos.edu.co - https://orcid.org/0000-0001-6797-9944

$\neq$ Administrador de Empresas, Universidad de los Llanos, Villavicencio, Colombia; joven investigador, Grupo de Investigación GYDO. andrey.laverde@unillanos.edu.co - https: //orcid.org/0000-0002-7925-3005

Criterio Libre N. ${ }^{\circ} 33$ Bogotá (Colombia) Julio-Diciembre 2020 pp. $111-125$ ISSN 1900-0642 ISSN electrónico 2323-0886 


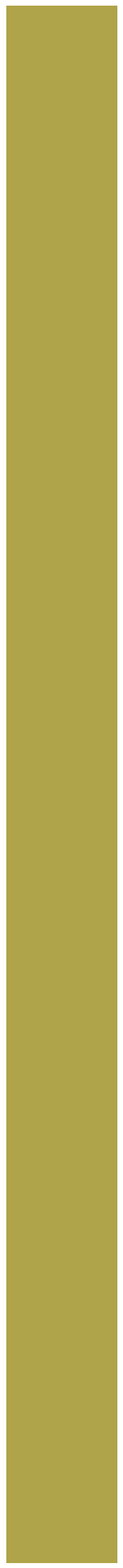

importante que resulta ser que el personal encargado de cuidar y velar por la salud de las personas esté motivado y se sienta bien en su trabajo, pues de esa manera prestará un mejor servicio. Para ello se realizó un estudio de tipo descriptivo con un enfoque cuantitativo, haciendo uso de los cuestionarios estructurados MPO5a y MPO5b y aplicándolos a los encargados de gestión humana en las IPS de la ciudad. Se obtuvieron resultados que aportan a una mejora en los procesos.

PALABRAS CLAVE:

beneficios; compensaciones; competitividad; incentivos; salario; servicio al cliente.

CLASIFICACIÓN JEL: JEL. J31, M54.

\begin{abstract}
Carrying out an analysis of human management processes turns out to be a very beneficial exercise to contribute to the improvement of companies, since it allows us to know the current situation and determine what is being done wrong, therefore knowing the salary characteristics presented by the lending institutions of the IPS Health Service of Villavicencio allowed to know the salary and hiring elements that IPS applies to its collaborators, and in addition, to identify the benefits and incentives with which they try to motivate and retain their collaborators, and taking into account the important that it turns out that the staff responsible for caring for and ensuring the health of people is motivated and feels good in their work, because that way they will provide a better service. For which a descriptive study with a quantitative approach was carried out, using the structured questionnaires MPO5a and MPO5b, and applying them to those in charge of human management in the IPS of the city, obtaining results that contribute to an improvement in the processes.
\end{abstract}

Keywords: benefits; compensations; competitiveness; customer service; incentives; salary.

JEL classification: J3 1, M54.

RESUMO

Fazer uma análise da remuneração salarial é um exercício muito benéfico para contribuir para a melhoria das empresas, pois permite conhecer a situação atual e determinar se algo está sendo feito de errado e, portanto, conhecer essas características que apresentam as instituições que prestam serviços de saúde, a IPS, de Villavicencio, 
permitiu conhecer o salário e os elementos de contratação que aplicam a IPS a seus colaboradores, além de identificar os benefícios e incentivos com os quais pretendem motivar e reter aos seus colaboradores, e determinar se é levado em conta o quão importante é o fato de que o pessoal encarregado de cuidar e zelar pela saúde de seus colaboradores. O estudo também identificou os benefícios e incentivos com os quais eles pretendem motivar e manter seus funcionários, e determinou se eles levam em consideração a importância de que o pessoal encarregado de cuidar e cuidar da saúde das pessoas estejam motivados e se sinta bem com seu trabalho, já que dessa forma eles prestarão um serviço melhor. Para este fim, foi realizado um estudo descritivo com abordagem quantitativa, utilizando os questionários estruturados MP05a e MP05b e aplicando-os aos encarregados da gestão humana no IPS da cidade. Os resultados obtidos neste trabalho contribuem para uma melhoria dos processos.

Palavras-chave: atendimento ao cliente; benefícios; competitividade; incentivos; remuneração; salário.

Classificação JEL: JEL. J3 1 - M54.

\section{RESUME}

Faire une analyse de la compensation salariale est un exercice très bénéfique pour contribuer à l'amélioration des entreprises, car il permet de connaître la situation actuelle et de déterminer si quelque chose est mal fait et, par conséquent, de connaître ces caractéristiques qui présentent les institutions qui fournissent des services de santé, IPS, de Villavicencio, en Colombie, a permis de connaître le salaire et les éléments de contrat qui appliquent les IPS à leurs collaborateurs, en plus d'identifier les avantages et les incitations avec lesquelles ils ont l'intention de motiver et de retenir leurs collaborateurs, et de déterminer si elle est prise en compte combien important est le fait que le personnel chargé de prendre soin et de veiller sur la santé de leurs collaborateurs. L'étude a également identifié les avantages et les incitations par lesquels ils entendent motiver et retenir leurs employés, et a déterminé s'ils tiennent compte de l'importance pour le personnel chargé de soigner et de veiller à la santé des personnes d'être motivé et de se sentir bien dans son travail, car c'est ainsi qu'il fournira un meilleur service. Pour ce faire, une étude descriptive avec une approche quantitative a été réalisée, en utilisant les questionnaires structurés MP05a et MPO5b et en les appliquant aux responsables de la gestion humaine dans les IPS de la ville. Les résultats obtenus dans ce travail contribuent à une amélioration des processus.

Mots clés: avantages; compétitivité; incitations; rémunération; salaire; service à la clientèle.

Classification JEL: J3 1 - M54. 


\section{- INTRODUCCIÓN}

Las entidades prestadoras de servicios de salud (IPS), hacen parte de un sector que, más que generar beneficios económicos, aportan al bienestar de la sociedad, ya que son las encargadas de prestar los servicios necesarios para cuidar la salud de las personas. Esta es una de las razones por las cuales surge la necesidad de estudiar el área y los procesos que están enfocados en prestar el servicio. Por ello estudiar el nivel de compensación salarial de los colaboradores de las IPS de Villavicencio permite identificar características en los procesos y a partir de esto, fortalecerlas o generar estrategias que aporten a la mejora continua.

En un estudio efectuado en Villavicencio sobre salarios se encontró que no es del interés de las clínicas aplicar incentivos, menos aún en asuntos de salario emocional, con una periodicidad de pago cercana a 30 ○ 45 días después, debido a una crisis del sector que afectaba los flujos de caja, con contrataciones en términos de prestación de servicio significativas para el especialista en salud que le permiten trabajar en varias instituciones debido a la poca oferta de personal en la región (Torres-Flórez, CastroGarzón, y Cepeda, 20181. Esto lleva a indagar si estas situaciones persisten y cómo mejoran respecto a las condiciones laborales de las empresas en estudio.

Con la investigación se logra percibir las prácticas de compensación salarial que utilizan las IPS de Villavicencio que aportan información que les permita ser más competitivas frente a otros sectores, determinando cuáles son las tendencias que se presentan en estas organizaciones en aspectos de compensación salarial; a esto se suma el hecho de que Villavicencio es el centro de atención en salud para la Orinoquia. Además, se identificaron dentro de los procesos de gestión humana en las IPS de Villavicencio las características de los salarios, incentivos y beneficios sociales, y gracias a esto se logra conocer el estado del sector y la forma en que se puede mejorar con la intención de prestar un servicio de calidad y aportar a la calidad de vida de los colaboradores.

Para poder desarrollar la investigación se hizo uso de fuentes primarias y secundarias, cada una de las cuales aportó información muy importante; en el caso de las fuentes secundarias se consultó a Castaño, Montoya, Restrepo, Varela, Corral, entre otros, donde cada uno desde sus investigaciones y conocimientos ha aportado contenido importante para la investigación, que además fue complementada por los resultados obtenidos de la aplicación de los cuestionarios estructurados MPO5a (Compensaciones) y el MP05b (Compensaciones por cargo) (TorresFlórez, 2017), a 18 IPS de Villavicencio. Con la información obtenida se logró un aporte importante al estudio de competitividad salarial de Villavicencio realizado por el grupo de investigación Gestión y Desarrollo Organizacional - GYDO de la Facultad de Ciencias Económicas de la Universidad de los Llanos.

\section{Desarrollo}

Todo lo relacionado con lo que se debe pagar a un trabajador por la realización de sus funciones, es decir, establecer una política de remuneración aceptable y justa para ambas partes, colaborador y empleador, es de constante preocupación para todas las organizaciones, sin importar su tamaño (Castaño, Montoya, \& Restrepo, 2009). Y cómo no darle importancia al manejo de uno de los elementos más notables en la motivación de las personas, ya que una buena compensación salarial puede ser la razón principal de la productividad en las empresas, por lo cual es tan importante ser justos y claros con el manejo que se da a este proceso.

Los salarios son el centro de la relación entre empleados y empleadores, todas las personas dentro de una organización dedican tiempo 
y esfuerzo al cumplimiento de unos objetivos a cambio de recibir una justa retribución en dinero, que represente el intercambio fijado en el contrato laboral renovado cada año junto con el resto de derechos y responsabilidades (Corral, 2006).

El salario se puede considerar en diferentes formas, puede ser el pago por un trabajo, pero también el valor que se da a un trabajador en una organización, o incluso un medio para posicionar jerárquicamente a un individuo en una empresa (Corral, 2006). Sin embargo, también es de manera general un costo y una inversión; un costo, porque se ve reflejado en el posterior precio del bien o servicio que ofrece la organización, y una inversión, porque representa la aplicación de dinero al recurso más importante de las organizaciones, como lo es el recurso humano, que es fuente de innovación, aprendizaje y riqueza (Mora, 2008).

Según Castaño, Montoya y Restrepo (2009), la compensación total se compone de tres grandes elementos: la remuneración base, los incentivos y los beneficios. La remuneración base hace referencia al sueldo base o al salario, es decir, es la remuneración o pago que se hace de manera regular por una empresa a un trabajador por sus servicios; depende de las responsabilidades, el mercado, el desempeño a largo plazo y las competencias establecidas en un sistema de pago equitativo para ambas partes interesadas (Torres-Flórez, 2019).

Los incentivos son una parte variable del salario, que reconocen un resultado superior por parte de un trabajador a sus responsabilidades establecidas, y teniendo en cuenta que no es obligatorio superarse en el puesto de trabajo, se tiende a motivar a los trabajadores mediante estos incentivos; por tanto, también debe existir una formalidad en el establecimiento de estos (Mora, 2008). Los beneficios son los componentes no monetarios de la compensación total; los más comunes son las vacaciones, cesantías, los seguros de vida y la salud, los convenios, el plan de retiro, entre otros. Por lo general, brindar estos beneficios depende en gran medida del tipo de organización, el tipo de cargo y los niveles jerárquicos (Fuentes López, Contreras Bravo y González Guerrero, 2020).

Las compensaciones deben cumplir cuatro funciones fundamentales en el logro de objetivos organizacionales: alineamiento estratégico, equidad interna, competitividad externa y dirección del desempeño (Castaño, Montoya, \& Restrepo, 2009); el alineamiento estratégico hace referencia a la forma en que las compensaciones afinan la relación entre los colaboradores y la organización, da una idea de la importancia que esta tiene en el momento de facilitar el cumplimiento de las metas de la organización, la motivación de los colaboradores y el desempeño de ellos. Mientras que la equidad interna busca que los pagos salariales se realicen según el impacto que genere cada cargo en la organización; entonces se puede definir la equidad interna como la igualdad de salarios entre compañeros de trabajo, de posiciones con responsabilidad similares y que tienen un impacto significativamente grande en la satisfacción y clima laboral (Cáceres, 2014$).$

Para la competitividad externa es importante estar pendiente de la oferta laboral que se presenta en el mercado, ya que si no se paga lo que este ofrece o no se mantiene actualizado en los dinamismos que se presentan en las compensaciones, se corre el riesgo de perder o no conseguir el recurso humano que cumpla con las más altas expectativas de la organización (Varela, 2013). Y para la dirección del desempeño debe haber una relación estrecha entre este y los objetivos y metas de la organización, ya que si no se direcciona el desempeño de los trabajadores al cumplimiento de las metas de la organización, se está perdiendo la fuerza del recurso más importante (Castaño, Montoya, \& Restrepo, 2009).

Existen entonces condiciones internas y externas que impactan los sistemas de remuneraciones, la dinámica de las organizaciones, las tendencias 
de los negocios y los ciclos económicos a los que se enfrenta el sector de la economía en que se encuentra inmersa la empresa (Restrepo, 2009.). Por lo anterior, los directivos empresariales buscan retener el talento humano adecuado para la empresa mediante diferentes alternativas de compensación que cumplan con las expectativas de los empleados; para ello deben existir elementos de liderazgo que lleven, a través de las TIC, políticas que aporten a la construcción de buenas prácticas laborales (Gazca Herrera, Sánchez Hernández, Culebro Castillo, y Zabala Arriola, 20191 . De esta manera las expectativas apuntan hacia el desarrollo profesional, obtener mayores ingresos, tener mayor poder e influencia en la organización. En este orden de ideas, es importante que las empresas conozcan cuáles son las consecuencias, causas y efectos que se tienen con un programa de compensación salarial que trabaje en pro de los colaboradores y, por tanto, de la organización, para así poder medir su nivel de competencia en los ámbitos nacional e internacional.

\section{METOdOLOGÍA}

Se efectuó un estudio de tipo descriptivo para conocer detalladamente las características salariales de las IPS de Villavicencio, en donde, a través de un enfoque cuantitativo en el que se aplicaron cuestionarios estructurados, diseñados para recolectar toda la información necesaria y pertinente para generar los resultados y conclusiones necesarios para alcanzar la mejora continua.

Para lograr realizar la investigación se tuvieron en cuenta las instituciones prestadoras de servicios de salud, IPS, de Villavicencio, obteniendo una muestra de 18 IPS sobre una población total de 21, a las cuales se les aplicaron dos cuestionarios; como criterio de selección se realizó con las IPS más relevantes respecto a su tamaño de colaboradores en la ciudad, aplicando el cuestionario
MP05a que cuestionaba generalidades de las compensaciones en las IPS, y el MP05b que pretende conocer específicamente las compensaciones por cargo (Torres-Flórez, 2017); se aplicó a 235 colaboradores de estas organizaciones; esta información hace parte de la información recolectada para el estudio de salarios 2018 en Villavicencio, organizada por el grupo de investigación Gestión y Desarrollo Organizacional (GYDO) y se usó el método de escala de medición en el cual se relacionan los porcentajes con términos cualitativos (Ver la tabla 1).

Tabla 1. Escala de medición.

\begin{tabular}{|l|l|l|}
\multicolumn{1}{|c|}{ Porcentaje } & \multicolumn{1}{|c|}{ Escala 1 } & \multicolumn{1}{c|}{ Escala 2 } \\
\hline $0 \%-20 \%$ & Muy malo & Casi nunca \\
\hline $21 \%-40 \%$ & Malo & $\begin{array}{l}\text { Muy pocas } \\
\text { veces }\end{array}$ \\
\hline $41 \%-60 \%$ & Regular & Ocasionalmente \\
\hline $61 \%-80 \%$ & Bueno & Frecuentemente \\
\hline $81 \%-100 \%$ & Muy bueno & Casi siempre \\
\hline
\end{tabular}

Fuente: Elaboración propia.

\section{RESUltAdOS Y DISCUSIÓN}

Para el desarrollo de la investigación se tomaron establecimientos privados, donde la totalidad están situados en el área urbana de la ciudad de Villavicencio. Las zonas que tienen mayor presencia de IPS son la comuna 2, perteneciente a los barrios Barzal Alto y Bajo de la cuidad. En la figura 3 se evidencia la localización de las IPS.

El porcentaje de participación más alto lo tiene el cargo de profesional, con 59\%, seguido del cargo auxiliar, con $20 \%$, lo cual nos indica que por cada 3 profesionales se tiene un auxiliar, pero si sumamos el cargo 1 y el 2 nos daría un porcentaje de participación de 29\%, lo que nos indicaría que por cada 2 profesionales tenemos 1 auxiliar 01 asistente. 
Tabla 2. Cargos por categoría (1).

\begin{tabular}{|c|c|c|c|}
\hline Auxiliar & Asistente & Asesor operativo & Supervisor \\
\hline $\begin{array}{l}\text { Auxiliar de enfermería } \\
\text { Recepcionista }\end{array}$ & Recepcionista & $\begin{array}{l}\text { Tecnólogo en } \\
\text { radiología }\end{array}$ & Supervisor \\
\hline Técnico en pediatría & Administrativo & $\begin{array}{l}\text { Medicina } \\
\text { alternativa }\end{array}$ & Coordinador laboratorio \\
\hline Administrativo & Asistente & Higienista oral & \\
\hline $\begin{array}{l}\text { Laboratorio médico y } \\
\text { clínico }\end{array}$ & Laboratorio & Conductor & \\
\hline \multicolumn{4}{|l|}{ Aseo general } \\
\hline Vigilancia & & & \\
\hline
\end{tabular}

Fuente. Elaboración propia.

Tabla 3. Cargos por categoría (2).

\begin{tabular}{|c|c|c|c|}
\hline Profesional & Jefe primera línea & Administrador & Gerente \\
\hline Ortodontista & Jefe de talento humano & $\begin{array}{l}\text { Administrador de } \\
\text { empresas }\end{array}$ & Coordinador SIG \\
\hline Cirujano oral & $\begin{array}{l}\text { Jefe departamento } \\
\text { contable }\end{array}$ & $\begin{array}{l}\text { Directiva Adm. y } \\
\text { Ciencia }\end{array}$ & $\begin{array}{l}\text { Médico con especialización } \\
\text { y maestría }\end{array}$ \\
\hline \multicolumn{4}{|l|}{ Radiología } \\
\hline $\begin{array}{l}\text { Pediatría } \\
\text { Médico general } \\
\text { Psicología } \\
\text { Salud ocupacional } \\
\text { Terapeuta } \\
\text { Especialista en } \\
\text { rehabilitación } \\
\text { Fonoaudiología } \\
\text { Bacteriólogo } \\
\text { Administrador } \\
\text { Enfermera profesional }\end{array}$ & & & \\
\hline
\end{tabular}

Fuente: Elaboración propia.

De todos los cargos, el que trabaja menos de 6 días a la semana es el de profesional, en $72 \%$; el otro $28 \%$ sí trabajan 6 días a la semana y de los 9 cargos solo el cargo profesional trabaja de 2 a 4 días a la semana, con 55\% de su totalidad. Cabe resaltar que el cargo de profesional está muy expuesto a ser contratado por prestación de servicios, y por ende, trabaja tan pocos días en la organización; caso contrario ocurre con el cargo administrador, gerente y supervisor, que tienen contratos a término indefinido en su mayoría y, por tanto, laboran 6 días a la semana el $100 \%$.
Se encontró una distribución interesante en la jornada laboral, donde $68 \%$ de los empleados de las IPS de la ciudad de Villavicencio trabajan tiempo completo y 10\% son trabajadores de medio tiempo; curiosamente en el sector salud se encontró que $22 \%$ de los trabajadores tienen jornada laboral ocasional, es decir, son trabajadores que no están permanentemente en la organización y sus actividades son muy específicas; por ejemplo, un cirujano oral, a quien le programan realizar una cirugía y previamente le informan la fecha y la hora, en ese momento hace presencia en la organización pero al terminar la actividad puede marcharse. 
Tabla 4. Perfiles de los cargos y sus salarios (2018).

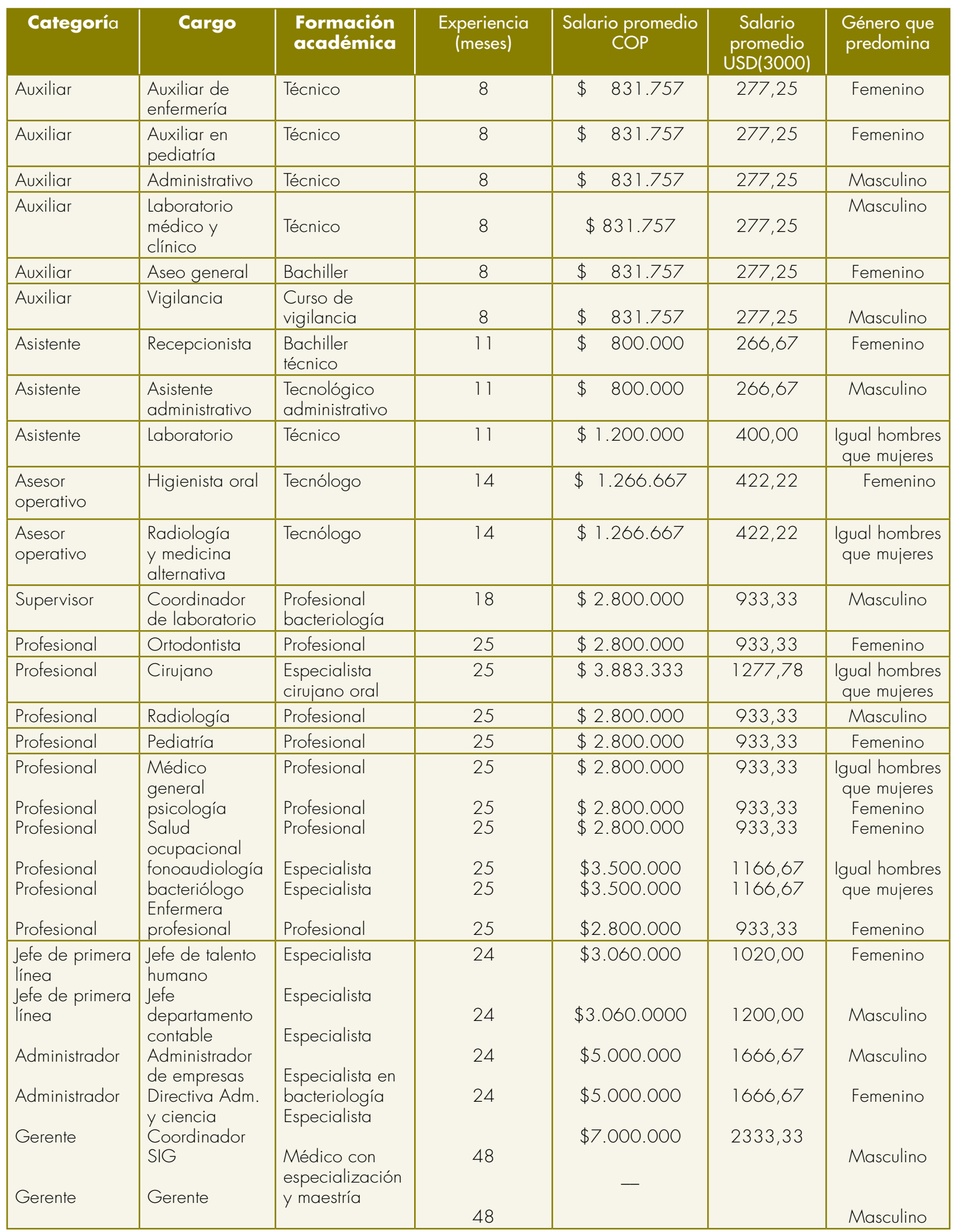

Fuente: Los autores. 
Luego de analizar los resultados de la formación académica en las IPS de Villavicencio podemos concluir que los salarios aumentan de acuerdo con el cargo; a mayor grado del cargo, el salario aumenta; cabe resaltar que en casos muy específicos esta función no se cumple; por ejemplo, se encontró que algunos profesionales suelen tener un salario más alto que los jefes de primera línea y esto sucede debido a que los jefes de primera línea son personal fijo, tienen su salario ya estipulado en el contrato laboral y aquellos profesionales suelen ser por prestación de servicios, lo que hace que el salario sea integrado y, por ende, al calcularlo mensualmente va a ser mayor. Hay que aclarar que muchos de los beneficios de los cuales goza el personal fijo no los tienen los de prestación de servicios.

Además, se puede evidenciar que en los primeros 4 cargos el máximo nivel académico es tecnólogo y el mínimo es bachiller, y por ello en el sector salud IPS de la ciudad de Villavicencio una persona que no tenga título de bachiller no puede aspirar a tener un cargo; en los últimos 4 cargos se puede evidenciar que en estos cargos el grado mínimo de educación es profesional y muchos tienen 2 o 3 especializaciones más, lo cual quiere decir que se tiene personal calificado para desarrollar efectivamente las actividades propuestas en la descripción de cargo.

En promedio la experiencia exigida para los primeros 2 cargos, es decir, asistente y auxiliar, es de 8 a 11 meses para aspirar a tener un cargo en el sector salud, para asesor operativo es de 14 meses y para supervisor, 18 meses de experiencia; a partir del cargo profesional en adelante hay una homogeneidad y la experiencia mínima debe ser de 24 meses, excepto para el gerente, que es de 48 meses.

El salario que devengan los asistentes y auxiliares no excede de 1 SMLV y los auxiliares operativos, en promedio 1,5 SMLV; los cargos de supervisor a jefe de primera línea devengan hasta 5 SMLV y los últimos 2 cargos devengan más de 5 SMLV, dependiendo de la empresa.
En promedio la experiencia exigida

para los primeros 2 cargos, es decir, asistente y auxiliar, es de 8

a 11 meses para aspirar a tener un cargo en el sector salud, para asesor operativo es de 14 meses y para supervisor, 18 meses de experiencia; a partir del cargo profesional en adelante hay una homogeneidady la experiencia mínima debe ser de 24 meses, excepto para el gerente, que es de 48 meses. 
Figura 1. Modalidad del salario.

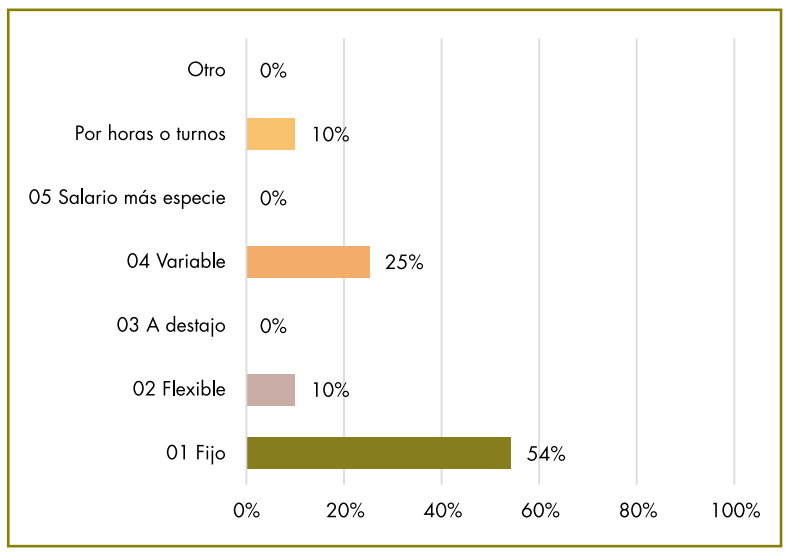

Fuente. Elaboración propia.

El $97 \%$ de la contratación se da de manera directa, es decir, sin intermediarios como agencias de empleo; hoy día es muy normal encontrar empleados contratados por bolsas de empleo y nunca tienen contratación directa con la organización, lo que disminuye los beneficios del empleado; cabe resaltar que solo por un tiempo de prueba este empleado puede ser contratado por intermediario y después de este período debe ser contratado directamente por la organización, o se estaría incumpliendo el Código Sustantivo del Trabajo (Código de trabajo, 2018); sin dejar a un lado el $3 \%$ de la contratación que se da en las IPS por medio de un tercero, con esta contratación se pudo identificar que se debe precisamente al período de prueba al que se expone al trabajador para luego ser contratado directamente por la organización.

El tipo de contrato que realizan las IPS de Villavicencio es 48, $1 \%$ indefinido y 30,4\% fijo, lo cual brinda tranquilidad a los trabajadores y, por ende, estos tienden a desarrollar eficientemente sus actividades; $21,5 \%$ restante se representa en un tipo de contrato de labor u obra, este claramente se presenta en personas que trabajan ocasionalmente en la organización, como lo hemos venido mencionando desde el inicio de la investigación. El hecho de estar estos trabajadores expuestos a este tipo de contrato no significa que sus salarios sean bajos. Por esto, no es un factor desmotivacional para ellos (Quezada y Torres, 2017), debido a que no es un factor desconocido para ellos puesto que están acostumbrados a prestar sus servicios de esta manera y con ello pueden laborar en varias entidades sin problema alguno (Figueroa, 2004).

La periodicidad del pago en la IPS de Villavicencio es quincenal o mensual, con $65,8 \%$ y $34,3 \%$, respectivamente; según Ramírez (2016), tanto los pagos quincenales como los mensuales son beneficiosos para las empresas ya que coinciden con los períodos contables; sin embargo, para los colaboradores el pago mensual resulta ser el menos conveniente, por el tiempo que deben esperar para recibir su pago.

Figura 2. Fecha de pago.

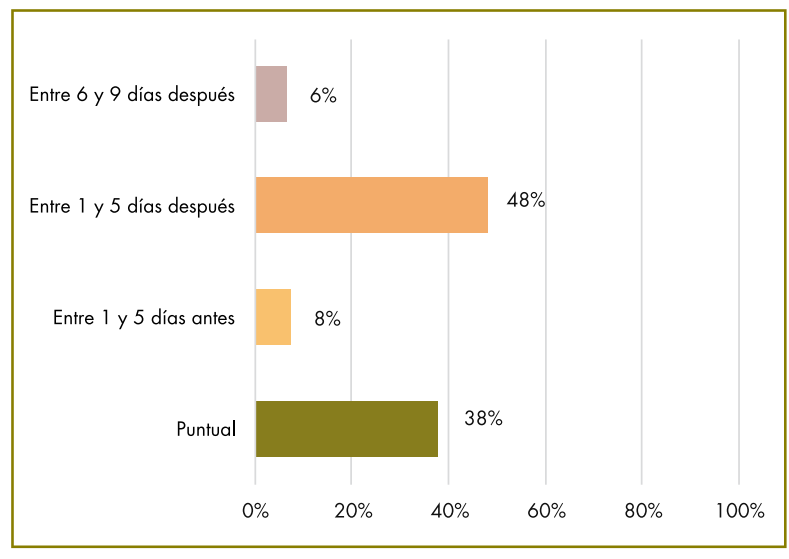

Fuente: Elaboración propia.

La forma de pago en las IPS de Villavicencio, en el $100 \%$ de los casos, es realizada por transferencia bancaria, es decir, todos los cargos deben contar con una cuenta de ahorros en la que se les deposita la nómina mensual - quincenalmente, según lo acordado. La importancia de implementar la forma de pago por transferencia bancaria es clara, gracias a esta forma de pago se puede evitar robos a la organización o equivocaciones en el momento de pagar; se evitan conflictos por desacuerdos en los empleados y se garantiza un buen clima organizacional (Castillo F. , 2012). 
Las IPS de la ciudad de Villavicencio no utilizan salario emocional, aunque conocen el concepto, y manifiestan no aplicarlo por órdenes de los cargos más altos y otras simplemente manifiestan estar trabajando en esto debido a que es un concepto nuevo para ellos. Ocurre que 100\% de las IPS no aplican salario emocional a sus trabajadores, también $100 \%$ de las organizaciones dicen conocer el concepto; evidentemente no es suficiente con conocerlo, también se debe saber qué es, cómo funciona, para qué sirve, aspectos positivos y negativos; luego sigue la implementación de este y no es suficiente con implementarlo, debemos estar revisando que sí se esté haciendo efectivamente, pues se sabe bien que las necesidades de cada persona son diferentes ya que unas pueden ser incentivadas por la parte monetaria, otras por tiempo de descanso, integraciones, formas de estudiar, etc. El salario emocional se ha convirtiendo en algo fundamental para aquellas organizaciones que buscan productividad, reducción del ausentismo y la rotación y un buen clima laboral (Gomez, 20111 ).

Figura 3. Veces que aplicaron aumento salarial en los últimos 4 años de modo general.

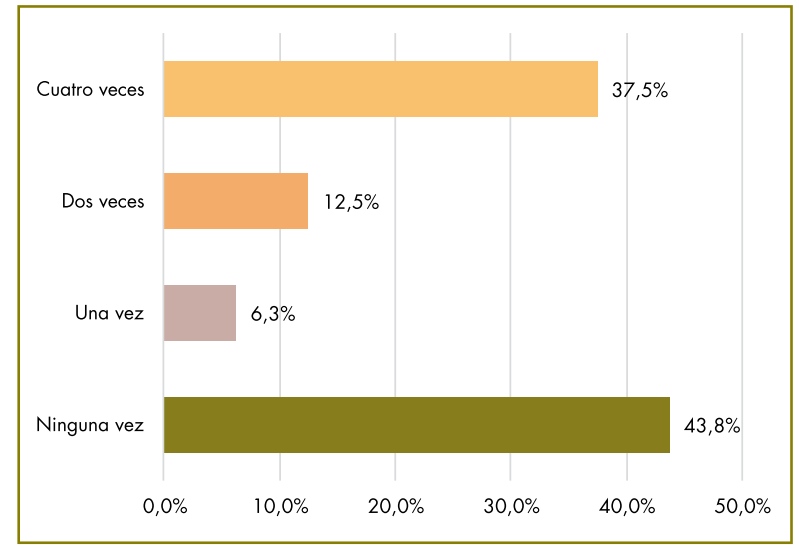

Fuente: Elaboración propia.

Los criterios que tienen en cuenta las IPS de Villavicencio para hacer aumento salarial son frecuentemente el porcentaje del SMLMV, con $65 \%$, y algunas veces por iniciativa de la empresa, 7,8\%, y otro, 4,7\%. Las IPS no tienen en cuenta la inflación y tampoco el porcentaje del IPC. Según (Gonzales A. , 2013), estos incrementos salariales resultan ser necesarios para retener a los buenos empleados, y en muchas ocasiones las empresas desprecian esta opción; esto es un grave error, pues es un factor que también mejora el ambiente en el sector ya que aspectos como la experiencia y competencias son igualmente parte de los elementos centrales en la toma de decisiones (Torres-Flórez, Castro-Garzón y Cepeda, 2018).

Según Jiménez (2013) a nivel interno de las organizaciones el aumento salarial se puede hacer sin regirse por ninguna normatividad; de hecho, no está legalmente establecida, cada empresa es autónoma en la manera como hace sus ajustes salariales; por esto las IPS de Villavicencio frecuentemente realizan aumentos salariales por cargos con 59\%, algunas veces con $13 \%$ por antigüedad y $9 \%$ en general a toda la empresa y $5 \%$ por áreas; es de resaltar la cifra de 59\% de aumento salarial por cargos, esta indudablemente se presenta debido a que los cargos más bajos como auxiliar y asistente no devengan más de un salario mínimo, por lo cual anualmente se les hace el aumento del salario mínimo como lo estipule la normatividad.

Figura 4. Incentivos que aplican las IPS.

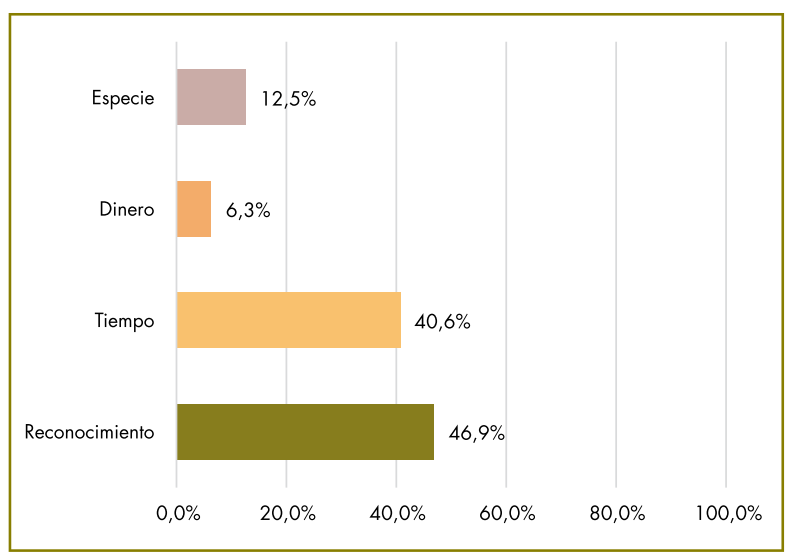

Fuente: Elaboración propia.

Las IPS de Villavicencio incentivan a los trabajadores basándose en ciertos criterios, entre ellos el más frecuente es el desarrollo de objetivos con un nivel de cumplimento de 36\%; desempeño 
El sector salud IPS de Villavicencio necesita efectuar el plan de mejora propuesto para pasar de ser un sector que conoce el concepto de salario emocional a ser uno que

lo implemente y le saque provecho para lograr ser así más competitivos frente a otros sectores. individual, con $36 \%$, y productividad, con $34 \%$; y en casos muy específicos utilizan otro tipo de criterio, con 3\% de participación. Son muy similares los porcentajes de participación de cada criterio, lo que significa que las IPS tienen muy en cuenta el desarrollo de los objetivos, pero de manera individual y considerando qué tan productivos sean para así mismo brindar incentivos a cada trabajador.

Figura 5. Beneficios sociales.

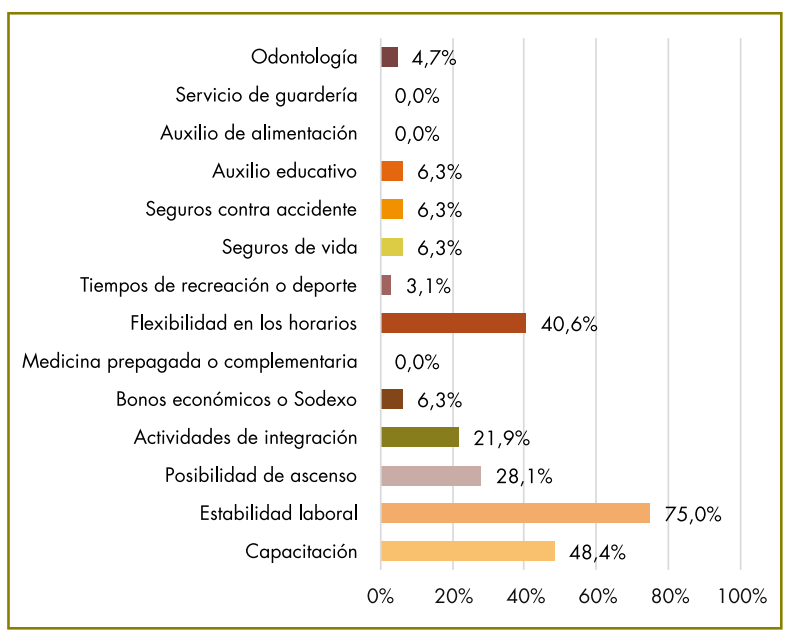

Fuente: Elaboración propia.

La evaluación de desempeño es algo que se debe desarrollar usualmente en cualquier tipo de organización, debido a que este proceso nos ayuda a ver en qué estamos bien y cómo podemos mejorarlo $y$, por otra parte, en qué estamos fallando ya sea a nivel individual u organizacional para así poder implementar estrategias a fin de contrarrestar estos errores. En las IPS de Villavicencio podemos observar que $50 \%$ de ellas hacen la evaluación de desempeño en forma mensual, $6,3 \%$ semestral y $6.3 \%$ trimestral; la parte alarmante es cuando encontramos que $37,5 \%$ de las IPS no realizan evaluación de desempeño.

En algunas empresas, y especialmente en algunos cargos, el género es un factor determinante, ya que de él depende la capacidad de cumplir o no con las exigencias del cargo, pero se evidencia 
que en las entidades prestadoras de servicios de salud IPS, ningún género sobresale en ninguno de los cargos, la diferencia más notoria está en los cargos de asistente y gerente, y se presenta más por la ausencia de determinado género frente al otro (Kotler, 2010).

En las IPS de Villavicencio la rotación de personal en el último año fue de 1 a 5 personas en 50\% de las IPS, $25 \%$ de 6 a 10 trabajadores al año, $12,5 \%$ de las IPS retiran más de 10 trabajadores al año, y en $12,5 \%$ de las IPS ningún trabajador se fue de la organización en el último año. Teniendo en cuenta que la estabilidad laboral es uno de los factores principales que brindan a los colaboradores, se creería que la cifra de rotación de personal sería menor, pero debemos tener en cuenta también el tipo de contrato puesto que se tiene un gran número de personal laborando por prestación de servicios, y además las IPS participantes en la investigación son de tamaño mediano y algunas grandes, y se conoce que, a mayor número de trabajadores, mayor es el número de retiros.

\section{CONCLUSIONES}

En las características salariales de las IPS de la ciudad de Villavicencio se logró evidenciar que el nivel de educación es fundamental para aspirar a un cargo en el sector, y también que los cargos que tienen mejor compensación salarial exigen un nivel de educación alto, es decir, como mínimo profesional, pero a su vez se evidencia que las IPS cuentan con personal capacitado y pueden ayudar a mejorar la productividad.

Las condiciones de contratación para las IPS en la ciudad de Villavicencio representan gran nivel de estabilidad laboral, debido a que las contrataciones se hacen a término indefinido o fijo; estos tipo de contratos le brindan cierto grado de tranquilidad al trabajador ya que este sabe que cuenta con su trabajo de tal fecha a tal fecha, en el caso del fijo, y el indefinido le brinda un mayor grado de confianza puesto que sabe que cuenta con el trabajo de manera fija y no está corriendo el riesgo de que sea retirado por terminación de contrato. Así mismo, al generar los contratos de manera directa, y no utilizando intermediarios como bolsas de empleo y/o empresas temporales que suministran personal a la hora de contratar, les permite a los colaboradores en el momento del contrato la posibilidad de negociar las condiciones y términos bajo los cuales va a desempeñarse, logrando adquirir mejores condiciones de empleo.

El sistema de incentivos y aumentos salariales que utilizan las IPS en la ciudad de Villavicencio presenta un grado de preocupación porque no están haciendo uso efectivo del salario emocional, aunque el concepto es conocido por las organizaciones; debido a esto se presenta el plan de mejora para ser implementado en las IPS buscando apostarle a ser más competitivos frente a otros sectores.

En cuanto a retribuciones económicas, las IPS aumentan el sueldo dependiendo de las labores; a mayor número de actividades, mayor sueldo; esto en casos específicos en contratos por labor u obra. Por otro lado, los incentivos en reconocimiento y tiempo son los que algunas veces utilizan las IPS, seguido de dinero y especie; por tanto, las IPS implementan algo de salario emocional indirectamente en la parte de reconocimiento y tiempo, pero se debe fortalecer para darle mayor grado de importancia y pasar de algunas veces a frecuentemente o siempre.

El sector salud IPS de Villavicencio necesita efectuar el plan de mejora propuesto para pasar de ser un sector que conoce el concepto de salario emocional a ser uno que lo implemente y le saque provecho para lograr ser así más competitivos frente a otros sectores. 


\section{RECOMENDACIONES}

Para las instituciones prestadoras de servicios de salud se recomienda definir políticas en las que se refleje la importancia de implementar el salario emocional, impulsar los incentivos de reconocimiento, tiempo, dinero y especie como eje para obtener mayor compromiso de los trabajadores. Desarrollar programas en los que se aplique evaluación de desempeño a los trabajadores de modo individual, grupal y organizacional, apoyar a los empleados para que se sigan formando académicamente, valorar los aportes y logros de los trabajadores, reconociendo sus esfuerzos y sus contribuciones, promover escenarios de esparcimiento para los trabajadores, definir a los trabajadores como parte primordial de la empresa, debido a que cada trabajador representa un papel importante para esta, y por eso hacerlos sentir felices hará que las IPS sean realmente prósperas y sostenibles. Incrementar la competitividad frente a otros sectores desarrollando actividades de recreación o deporte, con la finalidad de garantizar que se tengan empleados en un excelente estado anímico y así brindar una mejor atención al cliente, establecer un lugar de esparcimiento dentro de las IPS para los trabajadores en su tiempo libre.

\section{REFERENCIAS}

Cáceres, J. (2014). SCRIBD. Obtenido de SCRIBD: https://es.scribd.com/doc/57892536/ Historia-de-Las-Compensaciones

Castaño, C.; Montoya, L., y Restrepo, L. (2009). Compensación salarial y calidad de vida. Scientia Eł Technica, XV, núm.43. pp. 89-94.

Castaño, C.; Montoya, L., y Restrepo, L. (2009). Compensación salarial y calidad de vida. Obtenido de: https://dialnet.unirioja.es/ descarga/articulo/4603906.pdf

Castillo, PFW. (2012). El concepto de estrategia. blog.pucp. Recuperado de: http://blog.pucp. edu.pe/blog/freddycastillo/2012/03/14/ el-concepto-de-estrategia/

Código de trabajo (18 de 09 de 2018). leyes. co. Obtenido de: http://leyes.co/codigo sustantivo_del_trabajo/134.htm

Corral, F. (2006). Recursos humanos: Compensación. EOI Escuela de Negocios. Obtenido de: https://www.eoi.es/es/ file $/ 18662 /$ download? token=3J2NDXvM

Figueroa, AHA; Ramirez, VCA. (2004). México, D.F. Facultad de Filosofía y letras, Ciudad Universitaria, UNAMA. Obtenido de: http://ru.ffyl.unam.mx/bitstream/ handle/10391/4670/Admon_Serv

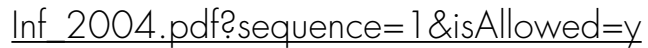

Fuentes López, H. J.; Contreras Bravo, L. E., y González Guerrero, K. (2020). Educación de la fuerza laboral en Colombia y sus efectos en los salarios. Revista Venezolana de Gerencia, 25(4), 364-376. Recuperado a partir de: https://produccioncientificaluz.org/index. $\mathrm{php/rvg/article/view/35197}$

Gazca Herrera, L. A.; Sánchez Hernández, G. L.; Culebro Castillo, K., y Zabala Arriola, O. (2019). Diagnóstico del uso de herramientas tecnológicas para la gestión y apoyo del liderazgo en las PYMES. Revista GEON /Gestión, Organizaciones Y Negocios), 6(11, 69-83. https://doi. org/10.22579/23463910.85

Gómez, C. (2011). Salario Emocional. Bogotá: Colegio de estudios superiores de administración. Obtenido de: https://repository.cesa.edu. co/bitstream/handle/10726/291/Bl47. pdf? sequence $=6$

González, A. (2013). Destino negocio. Obtenido de: https://destinonegocio.com/co/gestionco/la-productividad-es-fundamental-a-la-horade-un-incremento-salarial//

Jiménez, E. (2013). Compensación laboral y equidad interna. Bogotá. Obtenido de: 
https://repository.usergioarboleda.edu. co/bitstream/handle/11232/981/ Analisis $\% 20$ del $\% 20$ impacto $\% 20$ de $\% 20$ la\%20compensacion $\% 20 y \% 20$ beneficios $\% 20$ en\%20procesos. \%20gestion\%20humana. pdf? sequence $=1$ \&isAllowed $=y$

Kotler, P. (2010). Mercadotecnia. México: McGraw-Hill. Obtenido de: https://www. academia.edu/29671014/Marketing decimocuarta edici\%C3\%B3n Philip Kotler Gary_Armstrong.pdf

Mora, GNA. (2008). ¿̇Es la gestión del talento humano un factor de competitividad en las Pymes en la ciudad de Pasto? Revista Unimar, 26(2), 29-32. Obtenido de: http://editorial. umariana.edu.co/revistas/index.php/unimar/ article/view/86

Quezada Valadés, M. G., y Torres López, T. M. (2017). Representaciones sociales de la motivación laboral y la productividad de los trabajadores de la salud. La Piedad, Michoacán, México. Revista Salud Bosque, 711), 33-45. https://doi.org/10.18270/rsb. v7il.2093

Ramírez, E. (2016). MSCPayroll. Obtenido de: http://www.mscpayroll.com/blog/frecuenciade-pago-ventajas-y-desventajas/
Restrepo, L. S. (2009). Compensación salarial y calidad de vida. Obtenido de: https: / /dialnet. unirioja.es/descarga/articulo/4603906.pdf

Torres-Flórez, D. (2017). Modelo de medición de procesos de gestión humana en PYMES. Villavicencio.

Torres-Flórez, D.; Castro-Garzón, H., y Cepeda, L. (2018) Elementos de salarios, aumentos e incentivos en las clínicas de la ciudad de Villavicencio (Colombia), como herramienta de mejoramiento. En: Moran BLC., Torralba FA y Flores AMA. Experiencias de Emprendimiento, BUAP, México. https://www. researchgate. net/publication/344293572 Elementos de salarios aumentos e incentivos en las clinicas de la ciudad de Villavicencio Colombia_como_herramienta_de mejoramiento

Torres-Flórez, D. (2019). Estrategia de compensaciones como herramienta de satisfacción laboral. Revista GEON (Gestión, Organizaciones y Negocios), 6(2), 4-9. https://doi.org/10.22579/23463910.181

Varela, R. (2013). Administración de compensaciones, sueldos, salarios y prestaciones. Pearson educación. Obtenido de: https://cucionline.com/biblioteca/files/ original/8b6446e4bc766d7b63f03b41704 74e49.pdf 